\title{
Nouvelles recherches sur le déferlement des lames
}

\section{New research on the breaking of waves}

\author{
PAR J. LARRAS

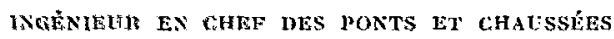

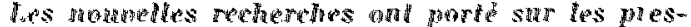

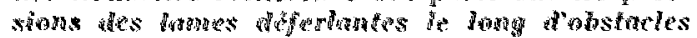

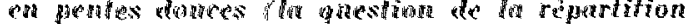

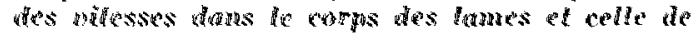

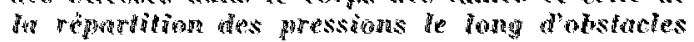

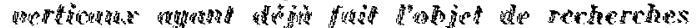

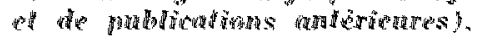

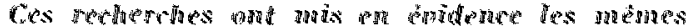

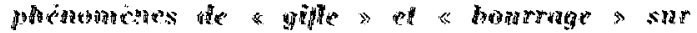

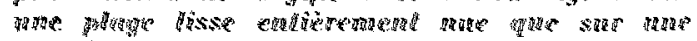

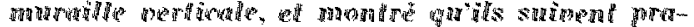

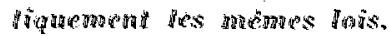

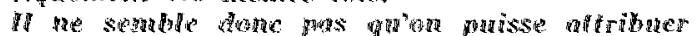

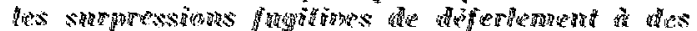

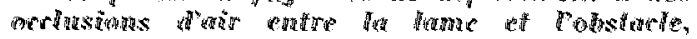

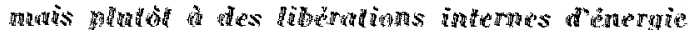

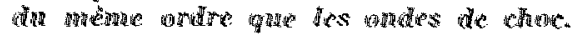

\begin{abstract}
The new research has been on the pressures cansed by manes breaking along obstacles of mild slope freserrct on the velocity distribution in the waves and the distribution of pressure along werital obstacles has already beon published:

This rearch has indicated the same short and long ferm "shock pressures" (" gifle" and "bourrage" on a smooth entirely mobstructed berreh as on wertical wall and has shomb that they follow prablically the same fows.

It does not seem therefore that the fugitigne onerparessures of the breaking whe ran be attributed to wis wapped between the wase wat Whe obstacle but jather to internal freeing of crergy of the stame order as shock menes.
\end{abstract}

Le diferlement des lames constitue l'un des pinenomenes tes phas dangereux, mais anssi les moins connus, quon pabse rencontrer dans la conception et daus la constructuon des ouvrages if himer.

Nous en axons poursuivi létude pendand phu-

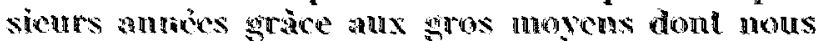

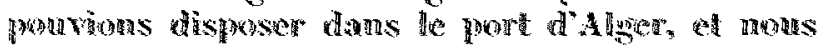

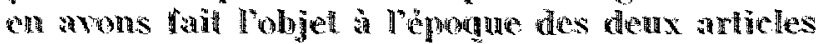
suivanalls :

- Le deferdonthent des lames sur les jelces ver-

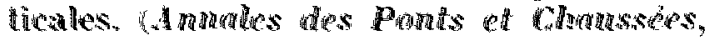
1987.)
- Recherehes expërimentales sur le déferlement des James. (Annales des Ponds ef Chanssies, 1952.)

Mais nous avons de hin dipuiser ee sujet difficile et notre suceesseur à la tête du port d'Alger, M. Ingenteur en ehef des Ponts et Chaussies Bauberune, a bien voula s'inmposer de ce fait Ia charge de poursurve nos budes plus avant, tout en nous permettant de eontinuer à les suive étroitement.

La prẻscule note a pour ohjel de rendre enmple du résultat de la nouvelle strie de recherches roubeprises minsi grace à lui. 


\section{争:}

Les recherches ont essentiellement porté sur les pressions des lames déferlantes le long d'obs. tacles en pente douce (la question des vitesses dans le corps des lames et celle des pressions sur les obstacles verticaux ayant déjà fait l'objet de recherches et de publications antérieures).

Elles ont en lieu an Laboratoire Central d'Hydraulique de France, avec le même générateur souple et dans le même canal vitré qu'au cours de nos essais précédents, la houle courant sur plus de 50 mètres par $0,60 \mathrm{~m}$ de profondeur avant de déferler sur une plage lisse à $7 \%$ de pente $\left(^{*}\right)$.

Elles ont comporté par ailleurs la mesure, pour 4 périodes de houle et pour trois types de cambrure différents :

- Des affleurements inférieurs et supérieurs et des niveaux médians ou de repos de la houle, en divers points, au moyen de limnimètres à palpeur électrique;

- Des pressions instantanées de la houle sur le fond, en 10 points de la zone de déferlement ou de ses abords, au moyen d'un manomètre à noyau magnétique ne faisant pas saillie sur la plage, d'un détecteur de variations d'inductance, et d'un enregistreur cinématographique à oscillographe cathodique.

$$
\text { ** }
$$

Nous avons trouvé en 1935-1936 que le « déferlement basculant $»$ sur une muraille verticale en travers d'une plage se traduit par :

$1^{\circ}$ Une pointe de pression extrêmement brutale

(*) La rugosité de la plage correspondait à $1 / c=0,019$ unités MKS, pour un rayon hydraulique de $0,07 \mathrm{~m}$, dans la formule de Tadini $\mathrm{U}=\mathrm{C} \sqrt{2} \mathrm{R} i$ qui régit l'écoulement uniforme à surface libre sur le même fond. et fugitive, d'origine élastique, que nous avons appelé la "gifle », tout au début du déferlement;

$2^{\circ}$ Une variation de pression moins brutale et plus continue, d'origine hydrodynamique, que nous avons appelée le "bourrage », pendant toute la suite du déferlement.

Nous avons retrouvé presque exactement la même chose en 1935 pour le * déferlement basculant » sur une plage lisse entièrement nue, à quelques différences près - qui n'ont pas d'importance fondamentale - dans l'allure générale des variations de la pression de «bourrage 》 en fonction du temps (voir fig. 1).

Nous avons trouvé en 1935-1936 que les pressions de «bourrage » du « déferlement basculant $»$ sur une muraille verticale en travers d'une plage ne dépendent pas beaucoup de la position exacte de cette muraille dans la zone de déferlement.

Nous avons retrouvé presque exactement la mème chose en 1953 pour les pressions de 《bourrage »du « déferlement basculant»sur une plage lisse entièrement nue: les pressions de «bourrage » ne dépendent pas beaucoup de la position exacte de l'observateur dans la zone de déferlement, et l'on note tout au plus qu'elles semblent comporter un léger maximum à l'aplomb du point où la lame commence à bouillonner avant d'aller s'écraser plus loin.

$$
\text { * }
$$

Vous avons trouvé en 1935-1936 que la différence des pressions de «bourrage » extrêmes dans le cas du "déferlement basculant» sur une muraille verticale en travers d'une plage n'atleint pas la hauteur d'eau qui correspond à la dénivellation maxima des lames dans Ia zone de déferlement.

Ainsi pour les pressions de «bourrage» au niveau de repos sur une muraille verticale en travers d'une plage lisse à $5 \%$ de pente :

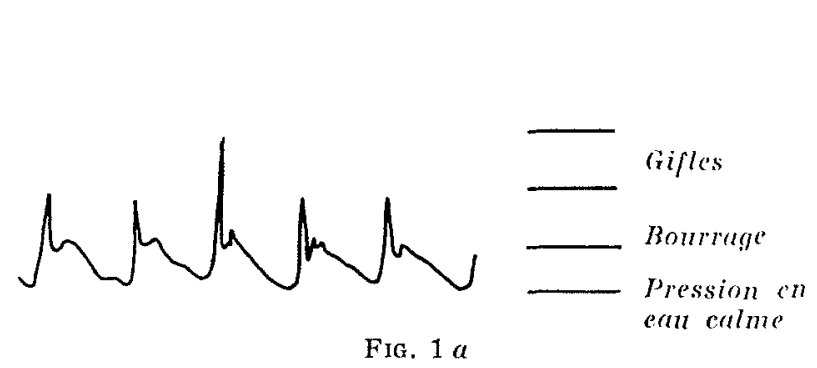

Pressions au fond de leau sur une plage Jisse entièrement nue à $7 \%$ de pente

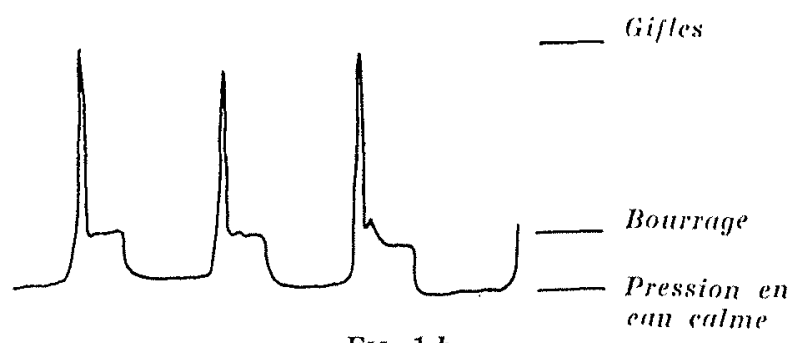

FIG. $1 b$

Pressions an niveau de repos sur une muraille verticale barrant une plage lisse at $10 \%$ de pente. 


\begin{tabular}{|c|c|c|c|c|}
\hline \multicolumn{4}{|c|}{ LAANES DÉFERLANTES } & \multirow{2}{*}{$\begin{array}{l}\text { Rapport } \\
\Delta p / \Delta h^{\prime}\end{array}$} \\
\hline $\begin{array}{c}\text { Périodes } \\
2 \mathrm{~T}\end{array}$ & $\begin{array}{l}\text { Denivella- } \\
\text { tions } \\
\text { maxima } \Delta h^{\prime}\end{array}$ & $\left\{\begin{array}{c}\text { Profondeurs } \\
\text { de: } \\
\text { deferlement* }\end{array}\right.$ & $\begin{array}{l}\text { Différences } \\
\text { des pressions } \\
\text { extrémest is } p\end{array}$ & \\
\hline s & $\mathrm{m}$ & $\mathrm{m}$ & m & \\
\hline 0,95 & 0,20 & 0,12 & 0,08 & 0,40 \\
\hline 1,10 & 0.27 & 0,16 & 0.10 & 0,37 \\
\hline 1,20 & 0,235 & $0,16^{2}$ & 0.115 & 0,49 \\
\hline 1,35 & 0,155 & 0,14 & 0,11 & 0,71 \\
\hline 1,65 & 0,23 & $0,10.5$ & 0,11 & 0,48 \\
\hline & & & & Moy : 0,49 \\
\hline
\end{tabular}

De mème pour les pressions de * bourrage au niveau de repos sur une muraille verticale en traters d'une plage lisse à 7,5 \% de pente :

\begin{tabular}{|c|c|c|c|c|}
\hline \multicolumn{4}{|c|}{ LAMES DLFERLANTES } & \multirow{2}{*}{$\begin{array}{l}\text { Rapport } \\
\lrcorner p / \Delta \hbar\end{array}$} \\
\hline $\begin{array}{c}\text { Periogestes } \\
\text { ? T }\end{array}$ & $\begin{array}{c}\text { Denivellat } \\
\text { Hors } \\
\text { maxima } \geq n^{*}\end{array}$ & $\begin{array}{c}\text { Profondeurs } \\
\text { de } \\
\text { diferlement" }\end{array}$ & $\left|\begin{array}{c}\text { Differrences } \\
\text { des pressions } \\
\text { extrèmes } \Delta p\end{array}\right|$ & \\
\hline$\$$ & nit & InI. & $\mathrm{m}$ & \\
\hline 0,95 & 0,20 & 0.155 & 0.08 & 0,31 \\
\hline 1.20 & 0,18 & 0,000 & 0,11 & 0,61 \\
\hline 1,20 & 0,185 & $0,1+1$ & 0.113 & 0,65 \\
\hline 1,20 & 0.28 & 0,15 & 0,12 & 0,52 \\
\hline 1,20 & 0,31 & 0.165 & 90,13 & $0,+12$ \\
\hline 1.5 .5 & A,, 25 & 41.12 & 0,12 & 0,48 \\
\hline
\end{tabular}

De mène enfin pour les pressions de bourrage an niveau de repos smr une muraille ver-

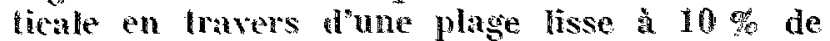
pente :

\begin{tabular}{|c|c|c|c|c|}
\hline \multicolumn{4}{|c|}{ LAMES DFFERLWWES } & \multirow{2}{*}{$\begin{array}{l}\text { Rapport } \\
\perp p \perp R^{*}\end{array}$} \\
\hline $\begin{array}{c}\text { Purriodes } \\
2 \text { T }\end{array}$ & 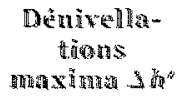 & $\begin{array}{c}\text { Protondeurs } \\
\text { dite } \\
\text { dererlemente" }\end{array}$ & 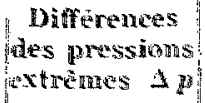 & \\
\hline$s$ & ant & inI & $\mathrm{m}$ & \\
\hline 1,95 & 41.175 & 4i, & 0,09 & 4,31 \\
\hline 1,10 & $n, 215$ & 0.905 & 0,095 & 0.44 \\
\hline 1.24 & $(0,235$ & 0,115 & 0.135 & 0,37 \\
\hline $1,3 \pi$ & a. & Ons & 0.12 & 7,73 \\
\hline \multirow[t]{2}{*}{155} & 0,15 & 0,00 & 0,125 & 0.83 \\
\hline & & & & \\
\hline
\end{tabular}

Nous arons retrouve presque exactement la mène elrose en 1953 pour les pressions de * hourrage * tu déferlentent basculan sur une plage lisse entièrement nue : Ia différence des pressions de bourrage exlremes au fond de leau natteint pas la hauteur d'ean qui cor-

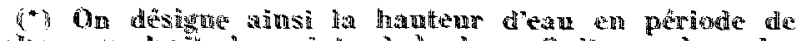

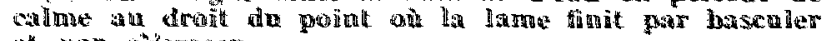

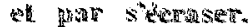

respond à la dénivellation maxima des lames dans la zone de déferlement.

Ainsi pour la pente de $7 \%$ :

\begin{tabular}{|c|c|c|c|c|}
\hline \multicolumn{4}{|c|}{ LAMES DÉFERLANTES } & \multirow{2}{*}{$\begin{array}{l}\text { Rapport } \\
\Delta p / \Delta h^{\prime}\end{array}$} \\
\hline Période: & $\begin{array}{l}\text { Dénivella- } \\
\text { tions } \\
\text { maxima } \Delta h^{\prime}\end{array}$ & $\mid \begin{array}{c}\text { Profondeurs } \\
\text { de } \\
\text { déferlement }\end{array}$ & $\left|\begin{array}{c}\text { Différences } \\
\text { des pressions } \\
\text { extrêmes } \Delta p\end{array}\right|$ & \\
\hline s & $\mathrm{m}$ & $\mathrm{m}$ & $\mathrm{m}$ & \\
\hline 1,2 & 0,048 & 0,046 & 0,0365 & 0,73 \\
\hline 1,2 & 0,076 & 0,055 & 0,055 & 0,72 \\
\hline 1,2 & 0,093 & 0,0725 & 0,063 & 0,70 \\
\hline 2,0 & 0,098 & 0,0925 & 0,070 & 0,71 \\
\hline 2,0 & 0,156 & 0,121 & 0,094 & 0,60 \\
\hline 2,0 & 0,188 & 0,143 & 0,118 & 0,63 \\
\hline 2,8 & 0,136 & 0,124 & 0,089 & 0,65 \\
\hline 2,8 & 0,160 & 0,140 & 0,111 & 0,69 \\
\hline 3,6 & 0,187 & 0,122 & 0,130 & 0,69 \\
\hline
\end{tabular}

Les ordres de grandeur que nous avons constatés en 1935-1936 concordent d'ailleurs tout à fait avec ceux de 1953 puisque la différence des pressions de «bourrage * extrêmes tout au fond de l'eau est de l'ordre de $0,50 \times(3 / 2)=0,75$ fois $\Delta h^{\prime}$ au pied d'une muraille verticale pour une plage lisse a 7,5 o de pente, d'après la figure 8 de notre article des Annales des Ponts et Chaussées de 1937 , alor's que nous avons trouvé 0,68 fois $\Delta h^{\prime}$ sans muraille verticale pour une plage lisse a $7 \%$ de pente en 1953 .

$$
x^{2}
$$

Nous avons trouvé en 1935-1936 que les dénivellations périodiques de l'eau près du point où la lame bascule et s'écrase sont répartis à raison denviron 3 au-dessus et d'environ $1 / 4$ au-dessous du niveau de repos.

Nous avons retrouré presque exactement la mème chose en 1953, non seulement pour les dénivellations périodiques de l'eau près du point où la lame bascule et s'ecrase, mais pour les variations simultanées de la pression de «bourrage sur le fond dans le cas d'une plage lisse entièrement nue.

Ia pression de * bourrage \$ maxima sur le fond est done de l'ordre de :

$$
+(3 / 4) \times 0,68 \Delta h^{\prime}=+1,02\left(\Delta h^{\prime} / 2\right),
$$

cest-î-dire du mème ordre que la dénivellation maxima par rapport au niveau médian près du point oi la lame basenle et s'écrase ( $)$.

La pression de kourrage minima est, de son côtét, de l'ordre de :

$$
-114 \times 0,68 h^{*}=-0,3+\left(\Delta h^{\prime} 2\right) \text {. }
$$

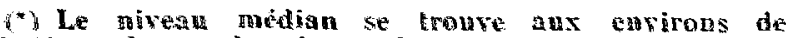

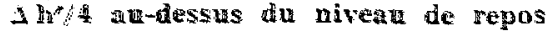


Et, comme on relìve des pressions sensiblement du même ordre dans le cas du déferlement sur une muraille verticale que dans le cas du déferlement sur une plage lisse entièrement nue, le «bourrage » du "déferlement basculant » ne présente, somme toute, pas plus de danger pour les ouvrages à la mer qu'un clapotis de même amplitude.

$$
* ;
$$

Nous avons trouvé en 1935-1936 qu'on ne relive de « gifles » appréciables sur une muraille verticale qu'au droit même du point où la lame bascule et s'écrase.

Nous avons retrouvé presque exactement la même chose en 1953 pour le « déferlement basculant $\gg$ sur une plage lisse entièrement nue : l'on ne relève de « gifles » appréciables sur le fond d'une plage lisse entièrement nue qu'au droit même du point où la lame bascule et s'écrase.

Les surpressions correspondantes varient donc beaucoup d'une lame à l'autre en un même point donné des murailles ou de la plage puisque la lame ne vient jamais s'écraser deux fois de suite au même endroit, ce qui modifie constamment le réglage extrêmement pointu de la courbe des pressions de la « gifle».

$$
* *
$$

Nous avons trouvé en 1935-1936 que la surpression maximum maximorum des « gifles » vagabondes vaut à peu près quatre fois la différence des pressions extrêmes du «bourrage» correspondant pour le « déferlement basculant»

\begin{tabular}{|c|c|c|c|c|c|}
\hline \multicolumn{5}{|c|}{ LAMES DÉFERLANTES } & \multirow{2}{*}{$\frac{\text { Gifle }}{\text { Bourrage }}=$} \\
\hline Pentes & Périodes & $\begin{array}{c}\text { Dénivella- } \\
\text { tions } \\
\text { maxima }\end{array}$ & Bourrage & Gifle & \\
\hline $\begin{array}{c}7,5 \% \\
7,5 \\
7,5 \\
10 \\
10 \\
10 \\
10 \\
10\end{array}$ & $\begin{array}{l}\text { mn } \\
0,95 \\
1,20 \\
1,20 \\
0,95 \\
1,10 \\
1,20 \\
1,35 \\
1,55\end{array}$ & \begin{tabular}{l}
\multicolumn{1}{c}{$\mathrm{m}$} \\
0,26 \\
0,18 \\
0,185 \\
0,175 \\
0,215 \\
0,235 \\
0,165 \\
0,15
\end{tabular} & \begin{tabular}{l}
\multicolumn{1}{c}{$\mathrm{m}$} \\
0,08 \\
0,11 \\
0,12 \\
0,09 \\
0,095 \\
0,135 \\
0,12 \\
0,125
\end{tabular} & $\begin{array}{l}\mathrm{m} \\
\\
0,30 \\
0,44 \\
0,46 \\
0,375 \\
0,46 \\
0,50 \\
0,50 \\
0,48\end{array}$ & $\begin{array}{c}3,75 \\
4,0 \\
3,85 \\
4,2 \\
4,85 \\
3,7 \\
4,15 \\
3,85 \\
\text { Moy. : } 4,0\end{array}$ \\
\hline
\end{tabular}
sur une muraille verticale en travers d'une plage:
Nous avons retrouvé presque exactement la même chose en 1953 pour le "déferlement basculant sur une plage lisse entièrement nue ("). Et, bien qu'il soit difficile d'aller très au-delà de cette simple indication que la pression de gifle vaut de deux à cinq fois la pression de bourrage, nous avons cru possible de dégager de nos nouvelles recherches les ordres de grandeur un peu

\begin{tabular}{|c|c|c|c|c|c|}
\hline \multicolumn{5}{|c|}{ LAMES DÉFERLANTES } & \multirow{2}{*}{$\frac{\text { Gifle }}{\text { Bourrage }}=$} \\
\hline \multirow[t]{2}{*}{ Pentes } & Périodes & $\begin{array}{l}\text { Dénivella- } \\
\text { tions } \\
\text { maxima }\end{array}$ & Bourrage & Gille & \\
\hline & $\mathrm{min}$ & $\mathrm{m}$ & $\mathrm{m}$ & $\mathrm{m}$ & \\
\hline $7 \%$ & 1,2 & 0,048 & 0,0365 & 0,15 & 4,1 \\
\hline 7 & 1,2 & 0,076 & 0,055 & 0,27 & 4,9 \\
\hline 7 & 1,2 & 0,093 & 0,065 & $0,19(?)$ & $2,9(?)$ \\
\hline & & & & & Moy : 4,0 \\
\hline
\end{tabular}
plus précis suivants :
La « gifle » du « déferlement basculant» semble donc présenter quatre fois plus de danger que le «bourrage », ou qu'un clapotis de même amplitude, pour les ouvrages à la mer.

(*) Notons toutefois que nous n'avons pas eu les moyens de faire varier les pentes de plages de part et d'autre de $7 \%$ dans les essais de 1953 .
Il parait être encore trop tôt pour pouvoir se rendre exactement compte des causes profondes et des lois générales du phénomène de la « gifle».

Il ne semble en tout cas dès maintenant plus possible d'en assimiler la surpression fugitive au choc (probablement élastique) de la masse d'cau de la lame déferlante, ou d'une masse d'air fugi- 
tivement occluse contre un obstacle qui devrait gêner cet épanouissement. Sinon l'on ne devrait pas relever de « gilles » au fond de l'eau dans le cas du «déferlement basculant $»$ sur une plage lisse entic̀rement nue.

L'on doit donc regarder plutôt le phénomène de la « gifle » comme un phénomène interne du même ordre que les ondes de choc dont toute libération brusque d’énergie s'accompagne générale- ment dans la nature, même en l'absence de tout obstacle.

Mais l'extrême difficulté d'une mesure certaine de la surpression maximum maximorum d'un phénomène aussi bref que vagabond que la 《 gifle »d'un « déferlement basculant » ne facilite certainement pas les choses aux chercheurs, et c'est pourquoi nous souhaiterions en voir augmenter très largement le nombre sur ce sujet délicat.

\section{DISCUSSION}

Président : M. WAHL

\section{Commentames de M. Miche :}

M. Miche signale d'abord que quelques-uns des faits indiqués dans la communication de M. LARras paraissent susceptibles d'unc interprétation theorique. Par oxemple :

$1^{\prime \prime}$ Il ressort du caleul des houles limites, en profondeur finie, guau début du déferlement, le rapport entre la surélération du niveau médian et la hauteur $\triangle \mathrm{h}^{\prime \prime}$ est proche de $1 / 4$ comme l'a trouve expérimentalement M. Lanfas. Toutefois, le rapport thëo-

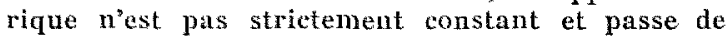
0,20 à 0,30 environ, lorsque la longueur de la houle, rapportie à la profondeur de déferlement, augmente.

$2^{\circ}$ La repartition des pressions, mesurées dans le cas d'une houle déferlante en profondeur décroissante, correspond assez sensiblement aux résultats donnés par la thérie courante de la houle en profondeur constante. Ceci montrerait que cette théorie de première approximation, toute sommaire qu'elle soit, conserverajt, a cet érard, sa validité dans ce cas limite et serait applicable, au moins a titre approche, au phẻnomène du « bourage».

$3^{\mathrm{k}}$ Si lon admet que les pressions beaucoup plus imporlantes de la $\ll$ gifle sont dues an choc de la lame deferlante s"écisant brutalement sur la plafre ef qu'il y anversion des quantités de monvement, c'est-a-dire choc èlastique, on obtient effecfivement des pressions comparables à celles enregistrées expérimentalement, et de lrordre de 4 fois celles correspondant a la diference des pressions de bourrage.

M. Mche considerant ensuite l'applieation des recherches de M. LARRAs au dimensiommement des ouvrages marilimes est conduit aux déductions suivantes :

La gifle, wa sa très courte durée et son aire localiséc at fuctuante a cours du temps, noparait pas comme un facteur determinant pour les ourrages dont les dimensions d'ensemble sont importantes vis-à-vis de l'amplitude de la houle. Elle justifie, néanmoins, l'adoption d'un certain coefficient de sécurité, à prendre en compte de toute facon pour de tels ourrages et, en outre, Femploi de matériaux résistant aux chocs répétés. Par contre, son influence relative doit croitre au fur et a mesure que les dimenstons de l'obstacle diminuent et ceel doit amener majorer dune facon plas importante les cfforts statiques agisssant sur lourrage. On sait que, dans certains phares, par exemple, des pièces métalliques de petite dimension ont été tordues sous l'effort considérable dr: la gifle.

En somme, il s'agit d'un phénomène qu'il y aurait intérêt à traiter sous l'angle statistique en vue de déterminer son importance relative en fonction des dimensions de l'obstacle rapportées à l'amplitude de la houle. Un coefficient de forme de lobstacle doit sans doute intervenir également.

Enfin, par son action très brutale, la gifle semble être un agent important de désorganisation des rivages, même en matériaux compacts, et, par conséquent, doit être un des facteurs responsables des transports littoraux.

\section{Discussion :}

M. Beaudevin craint que le maximum maximorum de la gifle ne soit difficile à saisir au moyen d'une mesure manométrique portant sur une surface qui n'est pas négligeable.

M. Mrche répond que l'appareillage utilisé permet d'enregistrer des pressions d'une durée de $1 / 100$ de seconde; il est possible que, pendant $1 / 10000$ de seconde, par exemple, la pression soit plus grande, mats pratiquement, ceci semble de peu d'importance.

En ce qui concerne les mesures faites en 1953 , les pointes de pression, relevées dans les limites perceptibles, sont bien définies et apparemment fort précises. Des pressions encore plus importantes ont été relevées a Dieppe « Etudes sur les efforts dus aux lames $\Rightarrow$, par MM. de Rouville. P. Besson et P. Petry, Annales des Ponts et Chaussées, 1938-VII) mais cette fois-ci en cours de déferlement; elles ont été attribuées à l'expansion brusque de l'air occlus; toutefois ce sont des phénomènes très fugaces qui n'avaient pas le caractère semipermanent des gifles.

Répondant à une question de M. LAcombe, M. Michs indique que les recherches de M. Larras permettraient le contrốle du critère de déferlement qu'il avait indiqué dans son étude de 1944 sur les Mouvements Ondulatoires de la Mer, mais qu'une vérification complète n'a pas été faite dans ce sens. Il rappelle que des études expérimentales effectuées il $y$ a quelques années au Laboratoire Dauphinois d'Hydraulique sur la forme limite de l'onde solitaire et des ondes de longueur courante études présentées au. Comité Technique - avaient donné l'oecasion de confronter, d'une manière satisfaisante, semble-t-il, les valeurs des cambrures des ondes limites mesurées avee celles données par le critère en question.

M. le Président remercie M. Mrche d'avoir présenté la communication de M. Larras et d'avoir donné des explications complémentaires. 